\title{
La vitamina D oral es efectiva para la prevención de fracturas no vertebrales en individuos de 65 años o más
}

\author{
Vitamin $D$ is effective in preventing nonvertebral fractures in persons older than 65 years old
}

Bischoff-Ferrari HA, y col. Arch Intern Med 2009;169(6):551-561.

\section{Objetivos}

Determinar la eficacia del suplemento oral de vitamina D (VD) en la prevención de fracturas en individuos de 65 años o mayores.

\section{Diseño}

Revisión sistemática y metanálisis de trabajos publicados en MEDLINE, EMBASE y Cochrane hasta agosto 2008, consulta con expertos en el tema y resúmenes presentados en ASBMR (American Society for Bone and Mineral Research).

\section{Selección de estudios}

Se incluyeron ensayos clínicos aleatorizados doble ciego que hayan evaluado la suplementación con VD oral (colecalciferol o ergocalciferol) con un seguimiento mínimo de un año, tuvieran más de una fractura en cada estudio, e indicaran adherencia y método de identificación de fracturas utilizadas. Se calculó la dosis recibida como producto de la dosis indicada y el porcentaje de adherencia.

\section{Resultados}

Se analizaron 12 estudios que cumplían los requisitos $(n=42.279$, edad media 78 años y $89 \%$ mujeres), de los cuales ocho $(n=47917)$ evaluaron fractura de cadera. En tres estudios la dosis de VD fue $<400 \mathrm{UI} / \mathrm{d}$ y en los nueve restantes, 482 a $770 \mathrm{UI} / \mathrm{d}$. En siete estudios se suplemento además calcio, 500 a $1200 \mathrm{mg} / \mathrm{d}$.
La duración del tratamiento osciló entre 12 a 84 meses. Los principales resultados se reportan en la tabla 1.

Tabla 1: Riesgo de fracturas según localización y dosis de suplementación de vitamina $D$.

\begin{tabular}{|c|c|c|c|c|}
\hline \multirow[b]{2}{*}{ Dosis VD } & \multicolumn{2}{|c|}{ Fracturas no vertebrales } & \multicolumn{2}{|c|}{ Fracturas de cadera } \\
\hline & $\begin{array}{c}\text { RR } \\
\text { (IC 95\% NNT) }\end{array}$ & $\begin{array}{c}\text { NNT } \\
\text { (IC 95\%) }\end{array}$ & $\begin{array}{c}\mathrm{RR} \\
\text { (IC 95\%) }\end{array}$ & $\begin{array}{c}\text { NNT } \\
\text { (IC 95\%) }\end{array}$ \\
\hline Cualquier dosis & $\begin{array}{c}0,86 \\
(0,77 \text { a } 0,96)\end{array}$ & & $\begin{array}{c}0,91 \\
(0,78 \text { a } 1,05)\end{array}$ & \\
\hline$<400 \mathrm{UI} / \mathrm{d}$ & $\begin{array}{c}1,02 \\
\text { (IC } 0,92 \text { a 1,15) }\end{array}$ & & $\begin{array}{c}1,09 \\
\text { (IC } 0,90 \text { a 1,32) }\end{array}$ & \\
\hline $400 \mathrm{U}$ & $\begin{array}{c}0,80 \\
\text { (IC } 0,72 \text { a 0,89) }\end{array}$ & $\begin{array}{c}93 \\
(66 \mathrm{a} \mathrm{160)} \\
\end{array}$ & $\begin{array}{c}0,82 \\
\text { (IC } 0,69 \text { a } 0,97) \\
\end{array}$ & $\begin{array}{c}168 \\
\text { (IC 104 a } 440 \\
\end{array}$ \\
\hline
\end{tabular}

En fracturas no vertebrales el uso de colecalciferol fue superior a ergocalciferol (23\% vs $10 \%$ respectivamente $p=0,07)$. El agregado de calcio no modificó los efectos observados. El efecto fue significativo en todas las edades consideradas y en individuos institucionalizados o no.

\section{Conclusiones}

La prevención de las fracturas no vertebrales es dosis dependiente. Dosis altas de VD podrían reducir las fracturas en al menos un $20 \%$ en individuos de 65 años o mayores.

Palabras claves: Vitamina D fracturas no vertebrales tratamiento. Key words: vitamin D nonvertebral fractures treatment.

Fuente de financiamiento: Fundación Nacional Suiza y Fundación Robert Bosch.

\section{Comentario}

Este estudio demuestra la importancia de la VD en la salud ósea. El tratamiento con la misma es barato seguro y está ampliamente disponible. Aún existe controversia sobre la diferencia de potencia entre ergocalciferol y colecalciferol y los estudios publicados son contradictorios ${ }^{1,2}$. En los últimos años las dosis recomendadas son más altas y si bien no existe consenso los expertos sugieren niveles séricos $>30 \mathrm{ng} / \mathrm{ml}$, determinando que una elevada proporción de individuos presente deficiencia y requiera suplemento. Los niveles mayores están relacionados con los efectos no clásicos de VD. Ellos son el rol en la actividad y fuerza muscular, secreción de insulina, mecanismos inmunitarios, antineoplásicos, y regulador de las funciones del sistema nervioso central y de la adipogénesis ${ }^{3}$.

El dosaje de VD tiene amplia variación metodológica y elevado costo. La medición basal es quizás la más importante porque permite calcular la dosis necesaria para suplementar. Se recordará que por cada $100 \mathrm{UI}$ aportadas la concentración sérica se eleva 0,5 a $1 \mathrm{ng} / \mathrm{ml}^{4}$. Las primeras evidencias de toxicidad son hipercalciuria, hipercalcemia e hiperfosfatemia. Los niveles tóxicos son $>150 \mathrm{ng} / \mathrm{ml}$.

La mayor fuente de la vitamina es la exposición solar. Son recomendables 5 a 30 minutos en hora pico dos veces semanales. Los ancianos por menor capacidad de producción cutánea deberán recibir 700 a 800 UI diarias ${ }^{4}$.

Los tenores adecuados también son necesarios en individuos jóvenes. Trabajos actuales demuestran mayor contenido mineral y masa muscular y menor tejido adiposo en niños y adolescentes con niveles óptimos ${ }^{5}$. Por lo tanto estimular su generación parece ser una medida sencilla para mejorar el capital óseo y contribuir a la disminución de fracturas en la vida adulta.

En el presente estudio la reducción de fracturas estuvo en relación directa con la concentración de VD y la dosis recibida. Si bien el descenso es discreto la relación costo-beneficio es favorable. En la práctica diaria existe alto nivel de incumplimiento resultante de información inadecuada, especialmente si el paciente recibe otras medicaciones potentes como bifosfonatos. Resta aclarar si mayor actividad física y mejor unidad musculo-esquelética tienen efectos sumatorios a la VD.

En conclusión todos los individuos con riesgo de fracturas o en presencia de ellas deben tener concentraciones séricas adecuadas de VD.

Ana María Galich [ Sección Osteopatías Metabólicas Servicio de Endocrinología Metabolismo y Medicina Nuclear del Hospital Italiano de Buenos Aires, ana,galich@ hospitalitaliano,org,ar ]

Galich AM. La vitamina D oral es efectiva para la prevención de fracturas no vertebrales en individuos de 65 años o más. Evid Act Pract Ambul. JulSet 2011;14(3):88. Comentado de: Bischoff-Ferrari HA Willett WC Wong JB y col. Prevention of Nonvertebral Fractures With Oral Vitamin D and Dose Dependency. A Meta-analysis of Randomized Controlled Trials. Arch Intern Med 2009;169(6):551-561. PMID: 19307517.

Referencias

1. Armas LA y col. Vitamin D2 is much less effective than vitamin D3 in humans. J Clin Endocrinol Metab. 2004;89 (11):5387-91.

2. Holick MF y col. Vitamin D2 is as effective as vitamin D3 in maintaining circulating concentrations of 25-hydroxyvitamins D. J Clin Endocrinol Metab. 2007;93(3):677-681.

2. Holick MF y col. Vitamin D2 is as effective as vitamin D3 in maintaining circulating
3. Holick MF. Vitamin D deficiency. Review article. N Engl J Med 2007;357:266-281.

4. Bikle D y col. Vitamin D: Production Metabolism Mechanism of Action and Clinical Requirements. Primer on Metabolic Bone Diseases and Disorders of Mineral Metabolism. Seventh

Edition. Editor-in-chief Clifford Rosen. Published by the American Society for Bone and Mineral Research, 2008;141-149.

5. Kremer R y col. Vitamin D status and its relationship to body fat final height and peak bone mass in young women. J Clin Endocrinol Metab 2009;94:67-73. 